\title{
FIRST DETECTION OF KRYPTON AND XENON IN A WHITE DWARF
}

\author{
KLaus Werner', Thomas Rauch ${ }^{1}$, Ellen Ringat ${ }^{\prime}$, and JefFrey W. KruK ${ }^{2}$ \\ ${ }^{1}$ Institute for Astronomy and Astrophysics, Kepler Center for Astro and Particle Physics, Eberhard Karls University Tübingen, Sand I. 72076 Tübingen, Germany \\ ${ }^{2}$ NASA Goddard Space Flight Center, Greenbelt, MD 20771, USA \\ Received 2012 May 2; accepted 2012 May 23; published 2012 ???
}

\begin{abstract}
We report on the first detection of the noble gases krypton $(Z=36)$ and xenon (54) in a white dwarf. About $20 \mathrm{KrVI}-\mathrm{VII}$ and Xe VI-VII lines were discovered in the ultraviolet spectrum of the hot DO-type white dwarf RE 0503-289. The observations, performed with the Far Ultraviolet Spectroscopic Explorer, also reveal highly ionized photospheric lines from other trans-iron group elements, namely Ga (31), Ge (32), As (33), Se (34), Mo (42), Sn (50), Te (52), and I (53), from which gallium and molybdenum are new discoveries in white dwarfs, too. For $\mathrm{Kr}$ and $\mathrm{Xe}$, we performed an NLTE analysis and derived mass fractions of $\log \mathrm{Kr}=-4.3 \pm 0.5$ and $\log \mathrm{Xe}=$ $-4.2 \pm 0.6$, corresponding to an enrichment by factors of 450 and 3800 , respectively, relative to the Sun. The origin of the large overabundances is unclear. We discuss the roles of neutron-capture nucleosynthesis in the-precursor star and radiation-driven diffusion. It is possible that diffusion is insignificant and that the observed metal abundances constrain the evolutionary history of the star. Its hydrogen deficiency may be the consequence of a late helium-shell flash or a binary white dwarf merger.
\end{abstract}

Key words: stars: abundances - stars: individual (RE 0503-289) - white dwarfs

Online-only material: color figures

\section{INTRODUCTION}

The spectroscopic detection of krypton and xenon in the universe is difficult because of their low cosmic abundance. In particular, they are not detectable in the Sun. The solar abundance values $(\log [N(\mathrm{Kr}) / N(\mathrm{H})]=-8.72 \pm 0.08$, $\log [N(\mathrm{Xe}) / N(\mathrm{H})]=-9.65 \pm 0.02$; corresponding to mass fractions $\log \mathrm{Kr}=-6.96$ and $\log \mathrm{Xe}=-7.78$; Lodders 2003) are based on meteoritic and solar wind isotope abundances as well as theoretical considerations of neutron-capture element systematics.

The krypton abundance in the interstellar medium (ISM) was determined by the analysis of $\operatorname{KrI} \lambda 1236 \AA$ line measurements. The derived value is $50 \%$ of the solar abundance (Cartledge et al. 2008) and this offset is not understood. The xenon abundance in the ISM is unknown:

Krypton and xenon were detected in planetary nebulae (PNe). The first analysis (Péquignot \& Baluteau 1994) of forbidden optical lines of $\mathrm{Kr} \mathrm{II}-\mathrm{V}$ and $\mathrm{Xe} \mathrm{II-IV}$ arrived at about 20 times solar abundances. That is interpreted as a consequence of $s$-process nucleosynthesis and dredge up in the PN progenitor stars.

The proof of krypton and xenon in stellar atmospheres was only successful in chemically peculiar stars. KrII and Xe II lines were discovered long ago (Bidelman 1962). Extreme overabundances, particularly for Xe (up to 5 dex), were found in $\mathrm{Hg}-\mathrm{Mn}$ stars (Dworetsky et al. 2008), being the consequence of radiative-driven diffusion.

Lines of highly ionized trans-iron group elements were discovered in hot subdwarfs $\left(T_{\text {eff }}=22,000-40,000 \mathrm{~K}\right)$ and white dwarfs (WDs, $T_{\text {eff }}=49,000-58,000 \mathrm{~K}$ ) by UV spectroscopy. $\mathrm{Ga}, \mathrm{Ge}, \mathrm{Sn}$, and $\mathrm{Pb}$ were identified in $\mathrm{sdB}$ and sdOB stars with enrichments up to 3 dex (O'Toole 2004; O'Toole \& Heber 2007). Vennes et al. (2005) found $\mathrm{Ge}$ in three $\mathrm{H}$-atmosphere WDs (spectral type DA). The average abundance is nearly solar. Chayer et al. (2005) detected Ge, As, Se, Sn, Te, and I in two He-atmosphere WDs (spectral type DO) namely $\mathrm{HZ} 21$
$\left(T_{\text {eff }}=53,000 \mathrm{~K}\right.$; Dreizler \& Werner 1996) and HD 149499B $\left(T_{\text {eff }}=49,000 \mathrm{~K}\right.$; Napiwotzki et al. 1995). The abundances range between 3 and 1000 times solar. It is unclear, to what extent this enrichment is the result of nucleosynthesis during the asymptotic giant branch (AGB) phase and/or the consequence of diffusion processes.

RE $0503-289$ is a hot DO-type WD $\left(T_{\text {eff }}=70,000 \mathrm{~K}\right.$; Dreizler \& Werner 1996). Spectra taken by the Far Ultraviolet Spectroscopic Explorer (FUSE) are extraordinarily rich in unidentified absorption lines that are not observed in any other WD. Considering the trans-iron group detections in the cooler subdwarfs and WDs, we may expect that many of the unidentified lines in RE 0503-289 also stem from these species, possibly being in higher ionization stages. Here we report on the discovery of $\mathrm{Kr}, \mathrm{Xe}$, and other trans-iron group elements and perform $\mathrm{Kr}$ and $\mathrm{Xe}$ abundance analyses.

\section{OBSERVATIONS AND LINE IDENTIFICATIONS}

We have combined FUSE spectra from three separate observations (M1123601, M1124201, P2041601), using procedures similar to those described in Werner et al. (2004), but with CalFUSE v3.2.3. The reductions of the two M112 observations were limited to time periods when the star was in the slit, and to orbital night; the net exposure time varied from $15 \mathrm{ks}$ to $16 \mathrm{ks}$, depending on instrument channel. For comparison with models, the spectrum was shifted to the photospheric rest frame. We are interested here neither in the light metals that we detected in the FUSE spectrum of RE 0503-289 (C, N, O, Si, P, S) nor in Fe and $\mathrm{Ni}$ in Hubble Space Telescope observations investigated by Dreizler (1999) and Barstow et al. (2000). We focus on trans-iron group elements.

\subsection{Krypton and Xenon}

Krypton $(Z=36)$. Within the FUSE wavelength band, 15 lines of $\mathrm{KrVI}$ are listed in the NIST ${ }^{3}$ database. Eleven of them are

3 http://physies.nist.gov/ 
Table 1

Utilized Spectral Lines of $\mathrm{Kr}$ and $\mathrm{Xe}$

\begin{tabular}{|c|c|c|c|c|c|c|c|c|c|}
\hline Ion & $\begin{array}{c}\lambda \\
(\AA)\end{array}$ & $\log g f$ & $\begin{array}{c}E_{i} \\
\left(\mathrm{~cm}^{-1}\right)\end{array}$ & $\begin{array}{c}E_{k} \\
\left(\mathrm{~cm}^{-1}\right)\end{array}$ & Configurations & Terms & $g_{i}$ & $g_{k}$ & $\begin{array}{c}\text { Identification } \\
\text { in RE 0503-289 }\end{array}$ \\
\hline \multirow[t]{14}{*}{$\overline{\mathrm{Kr} \text { VI }}$} & 919.93 & -1.966 & 170084 & 278787 & $4 s 4 p^{2}-4 p^{3}$ & ${ }^{2} S-{ }^{4} S^{0}$ & 2 & 4 & - (too weak) \\
\hline & 927.34 & -2.420 & 0 & 107836 & $4 s^{2} 4 p-4 s 4 p^{2}$ & ${ }^{2} P^{0}-{ }^{4} P$ & 2 & 2 & + \\
\hline & 931.39 & -2.005 & 8110 & 115479 & $4 s^{2} 4 p-4 s 4 p^{2}$ & ${ }^{2} P^{0}-{ }^{4} P$ & 4 & 6 & + \\
\hline & 944.05 & -0.959 & 170084 & 276011 & $4 s 4 p^{2}-4 p^{3}$ & ${ }^{2} S-{ }^{2} D^{\circ}$ & 2 & 4 & + \\
\hline & 956.61 & -0.974 & 222122 & 326657 & $4 s^{2} 4 d-4 s^{2} 5 p$ & ${ }^{2} D-{ }^{2} P^{\circ}$ & 4 & 4 & - (too weak) \\
\hline & 965.09 & -0.019 & 223040 & 326657 & $4 s^{2} 4 d-4 s^{2} 5 p$ & ${ }^{2} D-{ }^{2} P^{0}$ & 6 & 4 & + \\
\hline & 970.09 & -2.731 & 8110 & 111193 & $4 s^{2} 4 p-4 s 4 p^{2}$ & ${ }^{2} P^{0}-{ }^{4} P$ & 4 & 4 & + \\
\hline & 980.41 & -0.278 & 222122 & 324120 & $4 s^{2} 4 d-4 s^{2} 5 p$ & ${ }^{2} D-{ }^{2} P^{\circ}$ & 4 & 2 & + \\
\hline & 1002.75 & -2.746 & 8110 & 107836 & $4 s^{2} 4 p-4 s 4 p^{2}$ & ${ }^{2} P^{0}-{ }^{4} P$ & 4 & 2 & + \\
\hline & 1011.14 & -1.826 & 275380 & 374279 & $4 s^{2} 5 s-4 s 4 p 4 d$ & ${ }^{2} S-{ }^{2} P^{0}$ & 2 & 4 & - (too weak) \\
\hline & 1015.77 & -1.580 & 180339 & 278787 & $4 s 4 p^{2}-4 p^{3}$ & ${ }^{2} P-{ }^{4} S^{0}$ & 2 & 4 & + \\
\hline & 1045.23 & -0.823 & 180339 & 276011 & $4 s 4 p^{2}-4 p^{3}$ & ${ }^{2} P-{ }^{2} D^{0}$ & 2 & 4 & + (blend with O Iv) \\
\hline & 1052.95 & -1.561 & 183817 & 278787 & $4 s 4 p^{2}-4 p^{3}$ & ${ }^{2} P-{ }^{4} S^{a}$ & 4 & 6 & + \\
\hline & 1061.07 & -0.409 & 183817 & 278062 & $4 s 4 p^{2}-4 p^{3}$ & ${ }^{2} P-{ }^{2} D^{0}$ & 4 & 4 & + \\
\hline Kr vII & 918.44 & -0.134 & 170835 & 279715 & $4 s 4 p-4 p^{2}$ & ${ }^{1} P^{0}-{ }^{1} D$ & 3 & 5 & + \\
\hline \multirow[t]{9}{*}{$\overline{\mathrm{Xe}} \mathrm{vI}$} & 928.37 & -2.066 & 124870 & 232585 & $5 s 5 p^{2}-5 p^{3}$ & ${ }^{2} D-{ }^{4} S^{0}$ & 4 & 4 & + \\
\hline & 967.55 & -1.197 & 129230 & 232585 & $5 s 5 p^{2}-5 p^{3}$ & ${ }^{2} D-{ }^{4} S^{0}$ & 6 & 4 & + \\
\hline & 996.23 & $*-3.946$ & 0 & 100378 & $5 s^{2} 5 p-5 s 5 p^{2}$ & ${ }^{2} P^{\circ}-{ }^{4} P$ & 2 & 4 & - (too weak) \\
\hline & 1080.08 & $*-2.420$ & 0 & 92586 & $5 s^{2} 5 p-5 s 5 p^{2}$ & ${ }^{2} P^{\circ}-{ }^{4} P$ & 2 & 2 & + \\
\hline & 1091.63 & -2.005 & 15599 & 107205 & $5 s^{2} 5 p-5 s 5 p^{2}$ & ${ }^{2} P^{0}-{ }^{4} P$ & 4 & 6 & + \\
\hline & 1101.95 & -2.424 & 141837 & 232585 & $5 s 5 p^{2}-5 p^{3}$ & ${ }^{2} P-{ }^{4} S^{\circ}$ & 2 & 4 & + (blend with ?) \\
\hline & 1136.41 & +0.060 & 182308 & 270306 & $5 s^{2} 5 d-5 s^{2} 6 p$ & ${ }^{2} D-{ }^{2} P^{\circ}$ & 6 & 4 & + \\
\hline & 1179.54 & -2.731 & 15599 & 100378 & $5 s^{2} 5 p-5 s 5 p^{2}$ & ${ }^{2} P^{0}-{ }^{4} P$ & 4 & 4 & + \\
\hline & 1181.46 & -0.210 & 180250 & 264891 & $5 s^{2} 5 d-5 s^{2} 6 p$ & ${ }^{2} D-{ }^{2} P^{\circ}$ & 4 & 2 & + \\
\hline \multirow[t]{2}{*}{ Xe VII } & 995.51 & -1.496 & 0 & 100451 & $5 s^{2}-5 s 5 p$ & ${ }^{1} S-{ }^{3} P^{0}$ & 1 & 3 & + \\
\hline & 1077.12 & -0.555 & 143259 & 236100 & $5 s 5 p-5 p^{2}$ & ${ }^{1} P^{0}-{ }^{1} D$ & 3 & 5 & + \\
\hline
\end{tabular}

Notes. The last column denotes the (non-) identification in RE 0503-289. "indicates Xe vi $g f$ values that were adopted from the respective Kr vi lines.

detectable in RE 0503 -289 (Table 1, Figure 1). Three are too weak $(\lambda \lambda .919 .9,956.6,1011.1 \AA)$ and another one $(\lambda 918.1 \AA)$ is masked by a strong interstellar $\mathrm{H}$ I line.

There is no clear evidence for Krv lines. According to NIST, the two lines with highest relative intensity are located at $\lambda \lambda 965.09,1002.74 \AA$, and they fall almost exactly onto Krvi lines at $\lambda . \lambda 965.093,1002.746 \AA$. One may wonder whether this is a chance coincidence or a misidentification in the laboratory spectra. At any rate, a possible contribution of the $\mathrm{Kr} v$ to the KrvI lines is probably small. The KrvII line with the highest NIST intensity, $2918.44 \AA$, is detected (Figure 1). Grotrian diagrams of Kr VI and Xe VI are shown in Figure 2.

Xenon (54). Several of the Xevi lines listed in NIST are identified (Table 1, Figure 1). The two Xe vil lines with the highest relative intensity (NIST) are prominent: the resonance line $\lambda 995.51 \AA$ and $\lambda 1077.12 \AA$.

\subsection{Other Trans-iron Group Elements}

A quantitative analysis aiming at the abundance determination of the trans-iron group elements whose discovery is reported in this section requires laborious model atom construction and is beyond the scope of this Letter. Also, exhaustive line identifications must be deferred until spectrum synthesis calculations are performed. A few examples of the identified lines are shown in Figure 3.

Gallium (31). More than a dozen of the Gav lines from Kelly's (1987) line list ${ }^{4}$ are detected. This element was not detected before in a WD.

\footnotetext{
4 http://www.cfa.harvard.cdu/ampcgi/kelly.pl
}

Germanium (32). It is remarkable that all but one of the $23 \mathrm{Ge} v$ lines listed in NIST are present, plus five additional ones listed by Kelly (1987). Ge in that high ionization stage was never previously observed.

Arsenic (33). All four of the As v lines discovered by Chayer et al. (2005; see references for wavelength data therein) in HD 149499B are also present in RE 0503-289: $\lambda \lambda 987.65$, $1029.48,1051.6,1056.7 \AA$. We do not confirm the tentative identification of As IV $\lambda 999.3 \AA$ in RE $0503-289$ by Boyce et al. (2008).

Selenium (34). Se v $\lambda \lambda 1094.68,1151.0 \AA$ are detected as well as Se IV $\lambda \lambda 959.59,996.71 \AA$ (Chayer et al. 2005 wavelengths). Four lines of Se vi with wavelengths from Joshi \& van Kleef (1978) are seen, too: $\lambda \lambda 1056.98,1064.62$ (blend with PIV), $1080.69,1153.48 \AA$.

Molybdenum (42). NIST lists 10 Mo vI lines in the FUSE range. We detect the four lines with the highest relative intensity: $\lambda \lambda 995.811,1038.642,1047.184,1182.143 \AA$. Three of these have the largest $g f$ values. This element was not detected before in a WD.

$\operatorname{Tin}(50)$. Sn v $\lambda \lambda 1089.35,1132.79,1160.74 \AA$ (NIST) are detected, as well as Sn Iv $\lambda \lambda 1019.72$ (blend with Gav $\lambda 1019.71$ ), and $1044.49 \AA$ (Chayer et al. 2005).

Tellurium (52). The Te vi $\lambda \lambda 951.02,1071.40 \AA$ lines (wavelengths from Morton 2000) are rather prominent.

Iodine (53). We identify I vI $\lambda 1120.301 \AA$ (wavelength from Morton 2000).

\section{KRYPTON AND XENON ABUNDANCE ANALYSIS}

We computed NLTE model atmospheres (in hydrostatic and radiative equilibrium) with our TMAP code (Werner et al. 2003). 


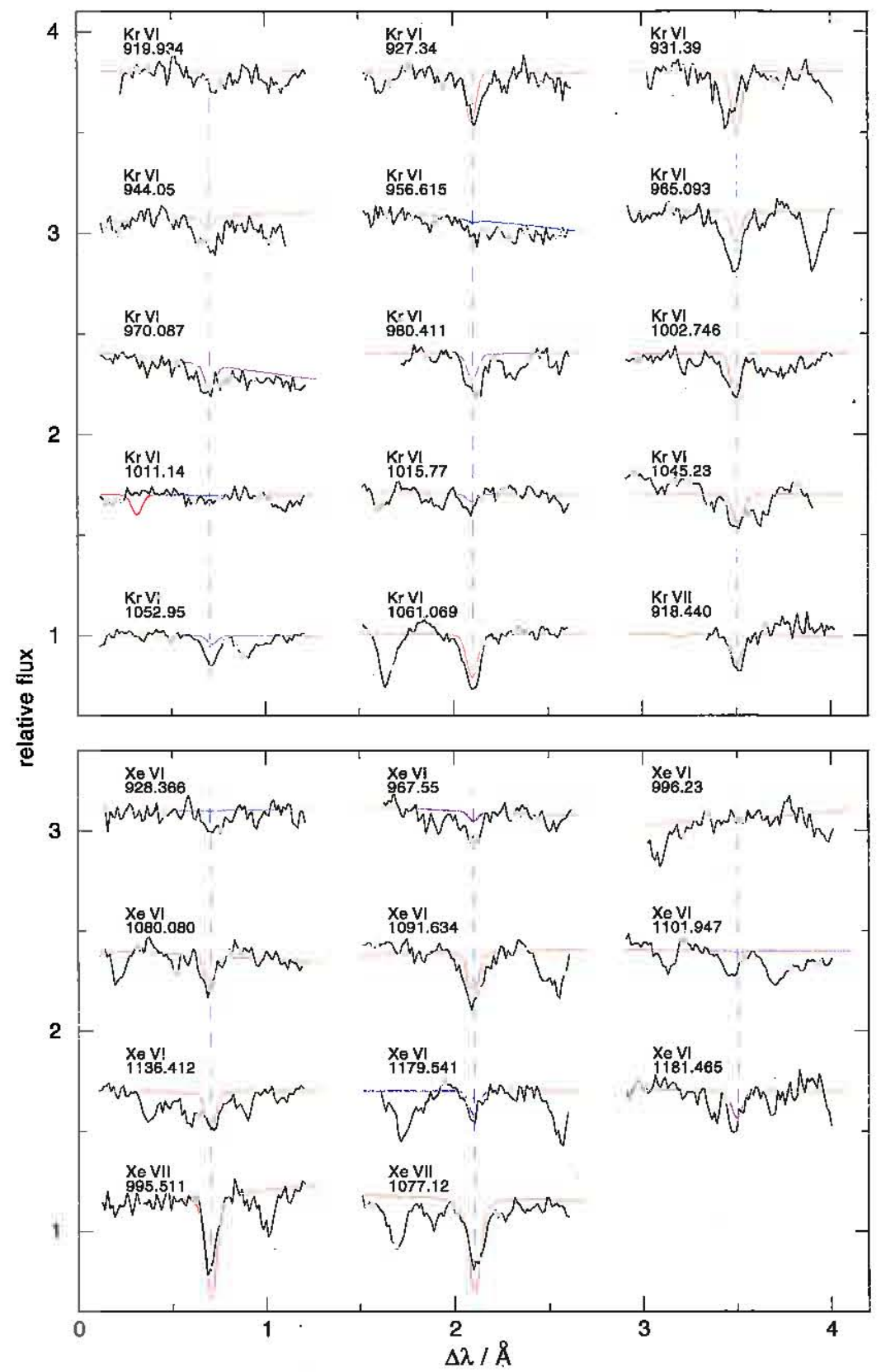

Figure 1. Top: Kr vI lines and a single Kr vII line (lower right profie) in RE 0503-289. Overplotted are computed profiles. Bottom: Xe vI and two Xe vil lines (two lowest profiles). The model atmosphere parameters are $T_{\text {eff }}=70,000 \mathrm{~K}, \log g=7.5, \log \mathrm{Kr}=\log \mathrm{Xe}=-4.2$ (mass fractions).

(A color version of this figure is available in the online journal.)

They are composed of $\mathrm{He}, \mathrm{C}, \mathrm{N}, \mathrm{O}, \mathrm{Kr}$, and $\mathrm{Xe}$, the latter two with various abundances. According to Dreizler (1999), we set to $\mathrm{C}=-1.7, \mathrm{~N}=-4.3, \mathrm{O}=-2.7$ (logarithm of mass fractions). $T_{\text {eff }}=70,000 \mathrm{~K}$ and $\log g=7.5$ were adopted from Dreizler \& Werner (1996). The model atoms used for this analysis were developed in the framework of the German Astrophysical Virtual Observatory ${ }^{5}$ project and are provided within the Tübingen Model-Atom Database (TMAD').

The Krvi model ion was constructed using NIST level energies that were compiled from Pagan et al. (1995) and

5 http://www.g-vu.org

6 http://astro.uni-tuebingen.de/ TMAD references therein. Oscillator strengths $f$ were taken from Pagan et al. (1996). Their list, however, is not complete which poses a serious problem for the NLTE calculations. $f$ values are unknown for 895 of the 1035 included line transitions. They were set to $10^{-4}$ within in a spin system and to $10^{-6}$ otherwise. It was found, however, that the resulting UV line profiles do not change when we completely omit them. In order to estimate the possible error of this procedure, we performed a test calculation in which the electron collisional rates were increased to very large values, such that LTE populations for all levels are enforced. As a result, the effect on the analyzed $\mathrm{Kr}$ lines is rather weak and the corresponding uncertainty in the abundance determination is well within the error estimated below. $f$ values for the lines in 

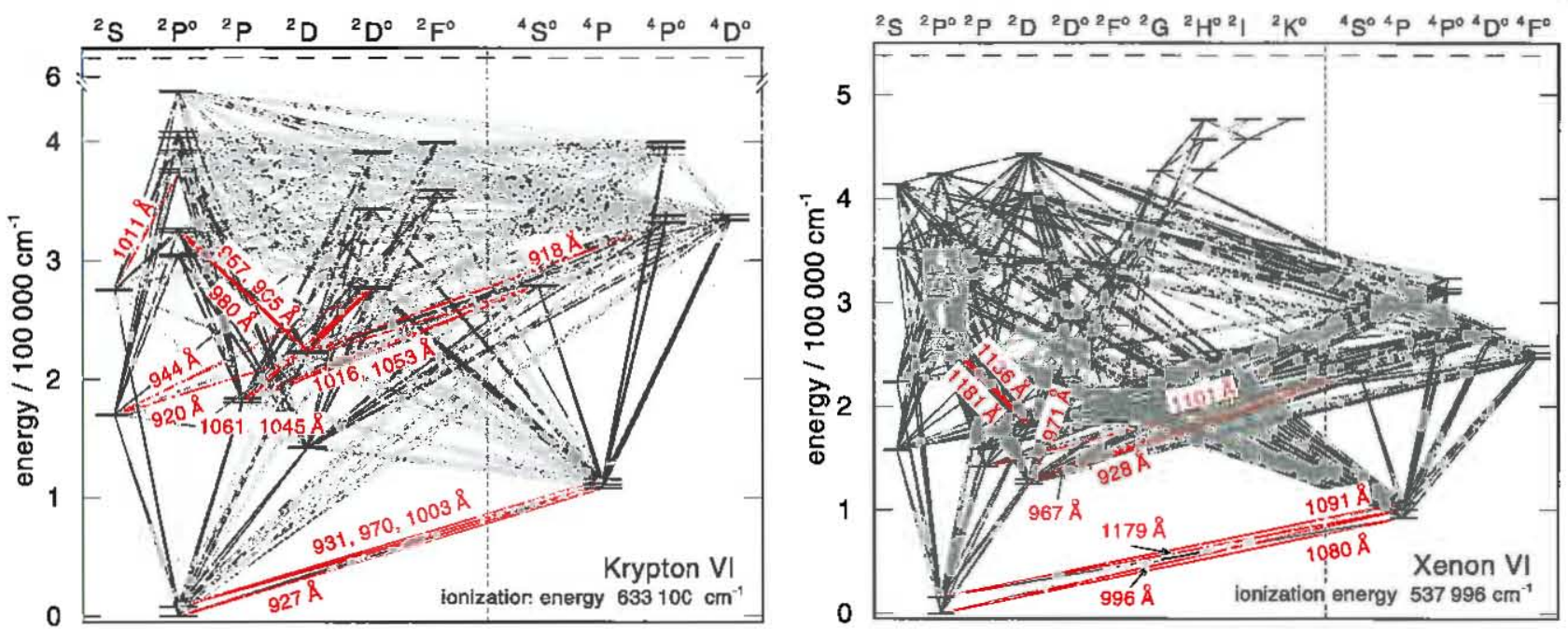

Figure 2. Grotriar diagrams of $\mathrm{KrvI}$ and $\mathrm{Xe}$ vI model ions. Dark and light gray graphs: radiative transitions with known and unknown $f$ values, respectively. Red graphs with wavelengths: spectral lines in the FUSE wavelength range ( $f$ values known).

(A color version of this figure is available in the online journal.)

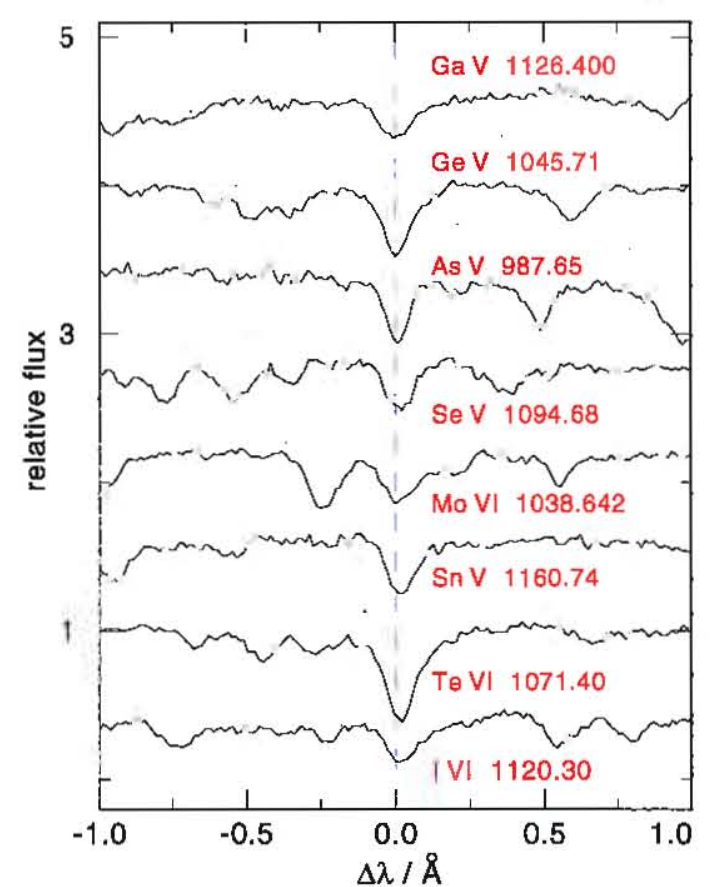

Figure 3. Examples of lines from a further eight trans-iron group elements identified in RE 0503-289.

(A color version of this figure is available in the online journal.)

the FUSE range are listed in Table 1. For Kr VII $\lambda 918.44 \AA$, we adopted the value of Victor \& Taylor (1983).

Photoionization rates were computed with hydrogen-like cross-sections. Electron collisional excitation and ionization rates were evaluated with usual approximation formulae following van Regemorter (1962) and Seaton (1962), respectively. For a proper ionization-balance computation, additional small models for neighboring ionization stages were constructed in order to complete the $\mathrm{Kr}$ model atom. In total, $\mathrm{KrII}-\mathrm{VIII}$ are represented by $1,38,25,46,14,1$ NLTE level(s), respectively.
The Xe vi model ion was constructed in a similar way, with level energies from Biémont et al. (2005). $f$ values were mainly taken from that source, too, and from Reyna Almandos et al. (2001). In total, $f$ values are unknown for 389 of the 887 included line transitions. NIST lists 16 lines in the FUSE range. For the line fit, we omit three weak ones that are blended by other strong lines, and another three because their NIST upper levels cannot be identified unambiguously with levels in Biémont et al. (2005). The remaining 11 lines used for our analysis are listed in Table I. Their $f$ values, if not available from Biémont et al. (2005), are taken from Reyna Almandos et al. (2001) or adopted from the respective $\mathrm{Kr}$ vi transitions. The $f$ values for the two observed Xe VII lines are from Kernahan et al. (1980) and Biémont et al. (2007). In total, our Xe III- virI model ions are represented by $1,44,29,82,36,1$ NLTE level(s), respectively. As for $\mathrm{Kr}$, we performed an LTE calculation and found a similar effect on the spectral lines.

The best line fits are achieved with $\log \mathrm{Kr}=-4.3 \pm 0.5$ and $\log \mathrm{Xe}=-4.2 \pm 0.6$ (mass fractions), i.e., 2.7 and 3.6 dex oversolar, respectively. It is obvious from Figure 1 that the quality of the line fits is rather diverse, some lines are reproduced too strong, others are too weak. Our error estimates are dominated by the corresponding uncertainties for the abundance determinations. In particular, the computed Xe VII lines come out too strong compared to the Xe vi lines. This could not be improved by reducing the temperature to $T_{\text {eff }}=65,000 \mathrm{~K}$.

\section{SUMMARY AND CONCLUSIONS}

We identified 10 trans-iron group elements in the hot DO RE 0503-289. Among them, $\mathrm{Kr}, \mathrm{Xe}, \mathrm{Ga}$, and Mo were never previously detected in WDs. For $\mathrm{Kr}$ and $\mathrm{Xe}$, we found extreme overabundances. Anticipating the results of future analyses, it is clear from the spectral appearance that the other trans-iron group species are strongly overabundant too. Taken together with the two cool DO WDs for which Chayer et al. (2005) have reported similar results, the $T_{\text {eff }}$ range in which this phenomenon occurs is now found to be $49,000-70,000 \mathrm{~K}$. Chayer et al. (2005) 
discussed that these overabundances might be ascribed to diffusion processes and to $s$-process nucleosynthesis in the AGB phase of the previous evolution. They also pointed out that this phenomenon is obviously restricted to DO WDs. This can be understood when considering that their immediate progenitors, the PG1 159 stars, also show signs of $n$-capture nucleosynthesis in the light-metal abundance patterns (Werner \& Herwig 2006). PG1159 stars expose their heavy-element enriched He envelopes which could serve as a reservoir from which high trans-iron element abundances can build up in the atmosphere by diffusion in the subsequent DO stage of evolution.

Chayer et al. (2005) found differences in the metal abundance patterns of their two cool DOs that are difficult to understand. Considering the similar values of $T_{\text {eff }}$ and $\log g$, diffusion should result in similar abundances unless differences in the pre-WD evolution had occurred.

Indeed there is evidence that at least two evolutionary channels produce DO WDs. The majority is going through the $\mathrm{H}$-deficient PG1 159 phase with high amounts of $\mathrm{C}$ and $\mathrm{O}$ in their He-dominated atmospheres, caused by a late $\mathrm{He}$-shell flash. There is a small number of so-called $\mathrm{O}(\mathrm{He})$ stars and related objects with similar atmospheric parameters but almost pure He-atmospheres (Rauch et al. 1998; Wassermann et al. 2010; Reindl 2012). We speculated that they originate from the RCrB stars, which themselves are possibly the product of a double WD merger (Saio \& Jeffery 2002). According to Unglaub \& Bues (2000), RE 0503-289 is still evolving toward the wind limit that must be crossed before diffusion overcomes radiationdriven mass loss. If this is true, then the surface abundances of RE 0503-289 are the outcome of a merger process with subsequent He-shell burning and $n$-capture nucleosynthesis. In this sense, RE 0503-289 might become an important validation check for yet-to-be-established evolution models. The observed abundance pattern could uncover details in the mixing and burning processes after the merger event. From AGB star models we know that trans-iron elements can be enriched in the $\mathrm{H}-\mathrm{He}$ intershell region by $\approx 2-3$ dex (Karakas et al. 2007; R. Gallino 2005 , private communication).

On the other hand, Unglaub \& Bues (2000) consider the possibility that RE $0503-289$ is the descendant of a PG1159 star that is a transition object passing through the wind limit, because the observed $\mathrm{C}$ abundance $(\approx 1 \%)$ is too small for a PG1159 star but too high to be explained by radiative diffusion. Any modeling attempt to explain the observed abundances of light and heavy metals would then have to account for both processes-mass loss and diffusion - in a manner that hitherto was only performed by Unglaub \& Bues (2000). In principle, radiative levitation can cause oversolar abundances as has been shown for metals as heavy as iron by Chayer et al. (1995).
We thank Emile Biémont and Pascal Quinet for providing Xe VI atomic data in electronic form. T.R. is supported by DLR grant 05 OR 0806 and E.R. by DFG grant WE1312/41-1.

Facility: FUSE

\section{REFERENCES}

Barstow, M. A., Dreizler, S., Holberg, J. B., et al. 2000, MNR: $:$, 314 , 109

Bidelman, W. P. 1962, A.J, 67, 111

Biémont, É, Buchard, V., Garnier, H.-P., Lefèbvre, P.-H., \& Quinet, P. 2005, Eur. Phrs. J. D, 33, 181

Biémont, É., Clar, M., Fivet, V., et al. 2007, Eur: Phys. J. I), 44, 23

Boyce, D. D., Barstow, M. A., Dobbie, P. D., et al. 2008, in ASP Conf. Ser. 391, Hydrogen-Deficient Stars, ed. K. Werner \& T. Rauch (San Francisco, CA: ASP), 235

Cartledge, S. I. B., Lauroesch, J. T., Meyer, D. M., Sofia, U. J., \& Clayton, G. C. $2008, A n J, 687,1013$

Chayer, P., Fontaine, G., \& Wesemael, F. 1995, ApJS, 99, 189

Chayer, P., Vennes, S., Dupuis, J., \& Kruk, J. W. 2005, .1pJ, 630, L16?

Dreizler, S. 1999, A\&A, 352, 632

Dreizler, S., \& Werner, K. 1996, A\&A, 314, 217

Dworetsky, M. M., Persaud, J. L., \& Patel, K. 2008, MNRAS, 385, 1523

Joshi, Y. N., \& van Kleef, Th. A. M. 1978, Physica B+C, 94, 270

Karakas, A. I., Lugaro, M., \& Gallino, R. 2007, A-J, 656, L73

Kelly, R. L. 1987, Atomic and Ionic Spectrum Lines below 2000 Angstroms, Hydrogen through Krypton (Melville, NY: AIP)

Kemahan, J. A., Pinnington, E. H., Neill, J. A., Bahr, J. L., \& Donnelly, K. E. 1980, J. Opt. Soc. Am., 70, 1126

Lodders, K. 2003, . ip I, 591, 1220

Morton, D. C. 2000 , InJS, 130,403

Napiwotzki, R., Hurwitz, M., Jordan, S., et al. 1995, A\&A, 300, LS

O'Toole, S. J. 2004, A\&. A, 423, L25

O'Toole, S. J., \& Heber, U. 2007, in ASP Conf. Ser. 372, 15th European Workshop on White Dwarfs, ed. R. Napiwotzki \& M. R. Burleigh (San Francisco, CA: ASP), 209

Pagan, C. J. B., Raineri, M., Bredice, F., et al. 1995, J. Opt. Soc. fim. B., 12, 203

Pagan, C. J. B., Raineri, M., Bredice, F., et al. 1996, J. Qu.nt. Spectros:., Radiut. Transfor, 55,163

Péquignot, D., \& Baluteau, J.-P. 1994, A\&A, 283, 593

Rauch, T., Köppen, J., \& Wolff, B. 1998, A\&A, 338, 651

Reindl, N. 2012, Master thesis, Eberhard Karls University, Tübingen

Reyna Almandos, J., Sarmiento, R., Raineri, M., \& Gallardo, M. 2001, J. Quant. Spectrozc. Radiat. Transfer, 70, 189

Saio, H., \& Jeffery, C. S. 2002, MNR. is, 333, 121

Seaton, M. J. 1962, in Atomic and Molecular Processes, ed. D. R. Bates (New York: Academic Press), 375

Unglaub, K., \& Bues, I. 2000, A\&A, 359, 1072

van Regemorter, H. 1962, ApJ, 136, 906

Vennes, S., Chayer, P., \& Dupuis, J. 2005, ApJ, 622, L121

Victor, G. A., \& Taylor, W. R. 1983, A.. Data Nucl. Dita Tables, 28, 107

Wassermann, D., Werner, K., Rauch, T., \& Kruk, J. W. 2010, Acc. i, 524, A9

Werner, K., Deetjen, J. L., Dreizier, S., et al. 2003, in ASP Conf. Ser. 288, Stellar Atmosphere Modeling, ed. I. Hubeny, D. Mihalas, \& K. Werner (San Francisco, CA: ASP), 31

Wemer, K., \& Herwig, F. 2006, PASP, 118, 183

Werner, K., Rauch, T., Barstow, M. A., \& Kruk, J. W. 2004, 1\& t, 421. 1169 


\section{Queries}

\section{Page 5}

Q1

Author: Please check the details for any journal references that do not have a pale purple link (CrossRef doi) or a blue link (NASA ADS or arXiv e-print) in the two-column proof (articlestyle layout). A journal reference with no links may contain some incorrect information.

\section{Online-only colour figures}

This proof PDF is identical in specification to the PDF file that will be published in the online journal. To view any online-only color figures as they will appear in the printed journal, we recommend that this color PDF file be printed on a black \& white printer. 